\title{
Highlights of the new 7th edition of the Canadian Tuberculosis Standards
}

\author{
Gale-Rowe $\mathrm{M}^{\mathbf{1}^{*}}$, Menzies $\mathrm{D}^{2}$, Sutherland $\mathrm{J}^{3}$ and Wong $\mathrm{T}^{1}$ on behalf of the editors and chapter authors \\ ${ }^{1}$ Public Health Agency of Canada, Ottawa, Ontario \\ ${ }^{2}$ McGill University, Montreal, Quebec \\ ${ }^{3}$ Canadian Thoracic Society/Canadian Lung Association, Ottawa, Ontario \\ *Corresponding author: Margaret.galerowe@phac-aspc.gc.ca
}

\section{Introduction}

In Canada, tuberculosis remains a significant health concern. The overall reported incidence of active tuberculosis cases continues to decline, but certain sub-populations, such as Aboriginal and foreign-born populations, are disproportionately affected. To date, dedicated research has allowed us to progress in our understanding of the pathogenesis, immunology, and epidemiology of tuberculosis as well as in the development of new diagnostic and therapeutic tools. This 7th edition of the Canadian Tuberculosis Standards (the Standards) has been extensively revised to incorporate this new information, building upon the six previous versions (1). Each chapter is written by authors from across Canada with expertise in the specific areas. The Standards are intended to provide best practice information but are not meant to replace consultations with healthcare professionals. They do not supersede any provincial or territorial legislative, regulatory, policy, and practice requirements, or professional guidelines that govern the practice of health professionals in their respective jurisdictions, whose recommendations may differ due to local epidemiology or context. This summary highlights some of the key changes in recommendations.

\section{Approach}

The Standards were co-produced by the Canadian Thoracic Society/Canadian Lung Association and the Public Health Agency of Canada in collaboration with the Association of Medical Microbiology and Infectious Disease Canada. As with previous editions, the 7th edition of the Standards is based upon the best available scientific evidence. Each chapter was developed by one or more authors with expertise in tuberculosis prevention and control. The authors of each chapter carefully reviewed all published evidence, particularly the most recent studies, and synthesized and rated this evidence using a modified Grading of Recommendations Assessment, Development and Evaluation framework (GRADE). Recommendations are considered strong or conditional on the basis of potential benefits, risks, and burden.

\section{Results}

The Standards contain important updates to best practices in tuberculosis prevention and control (Table 1). These include a new approach to sputum collection, whereby sputum specimens from tuberculosis suspects can all be collected the same day, potentially reducing the drop-out rate and improving yield. A new cartridge-based nucleic acid amplification test for detection of Mycobacterium tuberculosis is outlined. Available real-time polymerase chain reaction testing permits detection of $M$. tuberculosis and resistance in two hours. Results should be confirmed by routine smears and cultures. There are new recommendations on the appropriate use of the tuberculin skin test, and interferon gamma release assays (IGRAs), including when neither test is indicated.

There are major changes in the recommendations for both the schedule and the duration of therapy to treat tuberculosis, latent tuberculosis infection (LTBI), drug-resistant tuberculosis, and pediatric tuberculosis. Several practice recommendations are provided for drug-resistant tuberculosis. The need for routine HIV testing of all tuberculosis patients is emphasized; similarly, routine screening for latent tuberculosis infection is indicated in all patients with HIV infection. Due to the potential for major drug interactions, individuals with tuberculosis-HIV coinfection should be managed by, or in close collaboration with, a physician expert in tuberculosis-HIV care. In the healthcare setting, there are major changes in recommendations for baseline testing of healthcare workers, as 
well as new information on the risk of transmission. Changes in recommendations for the use of Bacille CalmetteGuérin (BCG) vaccine mean that BCG is no longer recommended for some groups, while for others use is on an exceptional basis only. In the area of contact follow up, details are provided on a change from the classic model to a prioritization of contacts based on characteristics of the source case and the susceptibility of the exposed.

\section{Table 1: Highlights of key changes to the 7th edition of the Canadian Tuberculosis Standards}

\begin{tabular}{ll}
\hline Chapter \\
\hline 1. & Epidemiology \\
\hline 2. & $\begin{array}{l}\text { Pathogenesis and } \\
\text { transmission of } \\
\text { tuberculosis }\end{array}$ \\
\hline
\end{tabular}

3. Diagnosis of active tuberculosis and drug resistance

4. Diagnosis of latent tuberculosis infection (LTBI) 5. Treatment of tuberculosis
disease
6. Treatment of latent tuberculosis infection (LTBI)

\section{Non-respiratory tuberculosis}

\section{Highlights and new recommendations}

Surveillance data provided up to 2010.

Probability of transmission and progression is described with specific populations.

New sputum collection approach consisting of three sputum specimens collected the same day with as little as one hour between specimens. Use of the diagnostic tool cartridge-based nucleic acid amplification test (NAAT) is outlined. Available real-time polymerase chain reaction testing permits detection of $M$. tuberculosis and resistance in two hours.

New recommendations on the tuberculin skin test (TST) and the interferon gamma release assays (IGRAs).

Major changes in recommendations for both the schedule of therapy and the duration of therapy. Information on drug doses for first- and second-line drugs, other intermittent treatment options, and tailored treatment for special populations.

Major changes in recommendations related to determining latent tuberculosis infection treatment, with recommendations for new shorter regimens, plus treatment of contacts of drug-resistant cases.

New information on sensitivity and specificity of diagnostic tests for different forms of extra-pulmonary tuberculosis and use of adjunctive steroids.

\section{Drug-resistant} tuberculosis

\section{Pediatric tuberculosis}

10. Tuberculosis and human immunodeficiency virus (HIV)

\section{Nontuberculous mycobacteria}

\section{Contact follow-up and outbreak management in tuberculosis control}

13. Tuberculosis surveillance and screening in selected high-risk populations
Major changes in treatment are recommended and ways to reduce drug resistance are outlined.

New information on risk groups and major changes in diagnosis and treatment.

Major changes in recommendations for treatment of latent tuberculosis infection and HIV as well as tuberculosis treatment in those requiring antiretroviral therapy (ART). Re-emphasizes need for routine HIV testing of all tuberculosis patients and routine screening for latent tuberculosis infection in HIV patients.

New recommendations identify when there is a need for an individual benefit/risk assessment regarding treatment.

A change from the classic concentric circle model to a prioritization of contacts looks at infectiousness of source, and the susceptibility of the exposed.

Identifies challenges and barriers to uptake of LTBI screening and treatment in select migrant populations. 


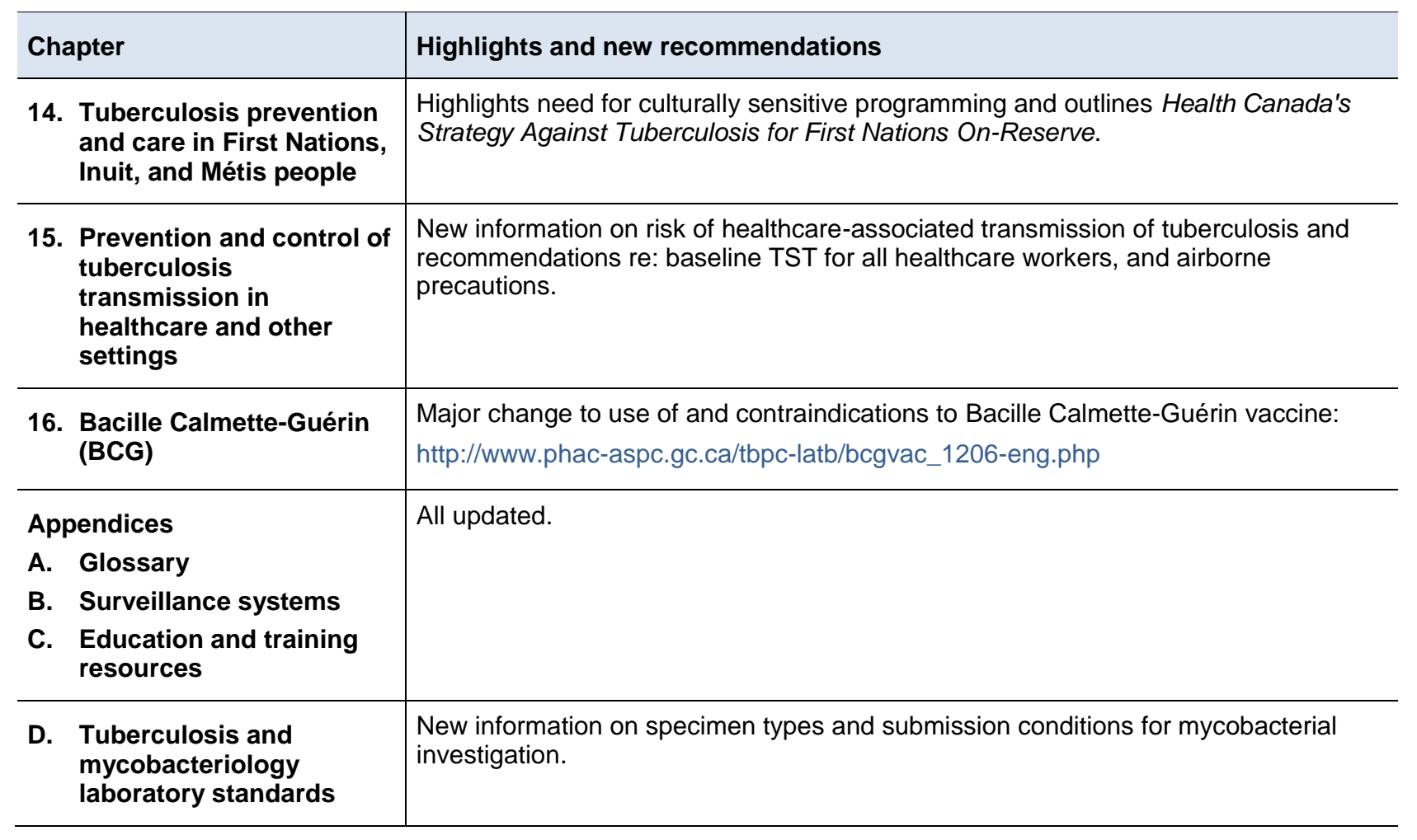

\section{Conclusions}

The Standards are the foundation for tuberculosis prevention and control in Canada. The 7th Edition highlights the contribution of new diagnostic and therapeutic tools to early diagnosis and successful case management, in the context of emerging antimicrobial resistance and co-infection.

\section{References}

(1) Menzies D, Wong T (eds). 2013. Canadian Tuberculosis Standards, 7th Edition. Canadian Thoracic Society, Canadian Lung Association, Public Health Agency of Canada. http://www.respiratory guidelines.ca/tbstandards-2014

\section{Acknowledgements}

Many thanks to the Editor, Dick Menzies and to the Associate Editors: Edward Ellis, Richard Long, Madhukar Pai, and Thomas Wong.

Many thanks to the chapter authors:

Alvarez GG, Archibald CP, Avendano M, Behr M, Christianson S, Cook V, Demers A-M, Ellis E, Elwood K, Embil J, Fisher D, Gallant V, Greenaway C, Halverson J, Houston S, Jamieson F, Jarand J, Khan K, Kitai I, Kunimoto D, Long R, Marras TK, Menzies D, Minion J, Ogunremi T, Orr P, Pai M, Rea E, Rivest P, Schwartzman K, Sharma $\mathrm{M}$, Wobeser WL, Wolfe,J, Wong, T. 


\section{Conflict of interest}

There are no conflicts of interest to declare.

\section{Funding}

This work was supported by the Public Health Agency of Canada. 